Editorial

\title{
Hip Arthroscopy in Femoroacetabular impingement
}

\section{Editorial}

Femoroacetabular impingement (FAI) is one of the commonest causes of hip pain. Also this resulted in limited hip range of motion especially in young adults and athletes. FAI has been divided into pincer, cam and combined types. The management is in both surgical and nonsurgical ways. In surgery, hip arthroscopy is the treatment of this disorder and also to treat associated injuries like labral tear etc. Compared to open surgeries arthroscopy has lower complication rates, less pain, and less morbidity. Nonsurgical treatment is by physiotherapy, strengthening of muscles and activity modification.

A recent review paper made an analysis and concluded that high percentage of patients return to sport activities after hip arthroscopy for FAI, with a low rate of complications and reoperation. All patientreported outcome measures, except for mental health, significantly improved after surgery. ${ }^{1}$

A systematic review done on the patient related improvement with positive outcome was based on factors such as younger age, male sex, lower BMI $\left(<24.5 \mathrm{~kg} / \mathrm{m}^{2}\right)$, Tonnis grade 0 , and those who got pain relief from preoperative intra-articular hip injections. Others who had negative outcomes were older age ( $>45$ years), female sex, longer duration of preoperative pain symptoms ( $>8$ months), elevated BMI, osteoarthritic changes, decreased joint space $(<2 \mathrm{~mm})$, chondral defects. $^{2}$

Another study done by $\mathrm{Kim}^{3}$ showed our meta-analysis revealed that arthroscopy was associated with superior iHOT-33 scores at the 12-month follow-up point. Except the iHOT-33 score, the clinical outcome scores investigated in the pooled studies revealed no differences between surgical and nonsurgical treatment of FAI. So they concluded there is no much difference in both treatments.

Finally for Femoroacetabular impingement (FAI) the treatment depends on various factors and the outcomes differ in different stages. Hip Arthroscopy plays a very important role and has better outcome. Nevertheless physiotherapy and exercises are to be done.

\author{
Volume 13 Issue 2 - 202 |
}

\author{
Nithin Sunku \\ Department of Orthopaedic Surgery, Global Hospital Trauma \\ Centre, India
}

Correspondence: Department of Orthopaedic Surgery, Global Hospital Trauma Centre, India, Email drsnithin@gmail.com

Received: December 16, 2020 | Published: March 29, 202 |

\section{Acknowledgments}

None.

\section{Conflicts of interest}

The authors declare no conflicts of interest.

\section{References}

1. Anas A Minkara, Robert W Westermann, James Rosneck Z, et al. Systematic Review and Meta-analysis of Outcomes After Hip Arthroscopy in Femoroacetabular Impingement. Am J Sports Med. 2019;47(2):488500.

2. Olawale A Sogbein, Ajay Shah, Jeffrey Kay, et al. Predictors of Outcomes After Hip Arthroscopic Surgery for Femoroacetabular Impingement. Orthop J Sports Med. 2019;7(6):2325967119848982.

3. Kim CH, Moon JK, Yoon JY, et al. Arthroscopy versus nonoperative treatment of symptomatic femoroacetabular impingement syndrome: a protocol for systematic review and meta-analysis. Medicine. 2020;99:49(e23247). 